\title{
Micrometer glass nozzles for flow focusing
}

\author{
J M Montanero ${ }^{1}$, A M Gañán-Calvo ${ }^{2}$, A J Acero ${ }^{1}$ and E J Vega ${ }^{1}$ \\ ${ }^{1}$ Department of Mechanical, Energetic and Material Engineering, University of Extremadura, \\ Avda. de Elvas s/n, E-06006 Badajoz, Spain \\ ${ }^{2}$ Department of Aerospace Engineering and Fluid Mechanics, University of Seville, \\ Camino de los Descubrimientos s/n, E-41092 Sevilla, Spain \\ E-mail: amgc@us.es
}

Received 11 February 2010, in final form 9 April 2010

Published 17 June 2010

Online at stacks.iop.org/JMM/20/075035

\begin{abstract}
We discuss the use of flame-shaped glass micro-nozzles for ultra-fine liquid atomization by flow focusing (DePonte et al 2008 J. Phys. D: Appl. Phys. 41 195505), which may have great importance in very varied technological fields, such as biotechnology, biomedicine and analytical chemistry. Some advantages offered by these nozzles over the original plate orifice configuration (Gañán-Calvo 1998 Phys. Rev. Lett. 80 285) are: (i) they are extraordinarily smooth even at the micrometer scale, (ii) one can readily obtain nozzles with neck diameters in the range of a few tens of microns, (iii) they demand gas flow rates significantly smaller than those required by the plate orifice configuration and (iv) they are transparent. However, highly demanding applications require a precise characterization of their three-dimensional shape by non-destructive means. This characterization cannot be obtained straightforwardly from optical transmission or electron microscopy mainly due to optical distortion. We propose in this paper a method for measuring the shape and size of micrometer nozzles formed inside millimetric and submillimetric capillaries made of transparent materials. The inside of the capillary is colored, and the capillary is put in a liquid bath with almost the same refractive index as that of the capillary to eliminate optical distortion. The nozzle image, acquired with a microscope using back-light illumination to get a silhouette effect, is processed to locate the contours of the nozzle with sub-pixel resolution. To determine the three-dimensional shape of the nozzle, the capillary is rotated in front of the camera. The method provides precise results for nozzle sizes down to a few microns.
\end{abstract}

(Some figures in this article are in colour only in the electronic version)

\section{Introduction}

The flow focusing (FF) method [1] is a continuous droplet production technique working in the so-called jetting mode $[2,3]$. A gas current induces steady-tip streaming in a liquid meniscus attached to a feeding capillary, and 'focuses' the microjet emitted from the meniscus tip across an orifice (figure 1). The microjet breaks up due to the growth of axisymmetric perturbations downstream (the Rayleigh instability $[4,5]$ ) leading to the formation of drops, which are commensurate with the jet diameter. FF has some advantages over other techniques for production of microdrops: (i) it uses purely hydrodynamic means to produce the drops; (ii) it is, therefore, applicable to any liquid; (iii) it allows one to obtain drops with diameters from $500 \mathrm{~nm}$ to $500 \mu \mathrm{m}$; (iv) it produces collections of drops with a high degree of monodispersity; (v) it can reach high production rates (up to $10^{6}$ drops s$^{-1}$ ); (vi) it can be adapted to a two-dimensional topology $[6,7]$.

The FF principle [1] was originally applied using the plate orifice (PO) configuration (figure 1). In this configuration, the gas stream co-flows with the liquid jet across an orifice of a plate located in front of the feeding capillary. The two main geometrical parameters of the fluid configuration are the capillary-to-orifice distance $H$ and the orifice diameter $D$, which essentially determines the gas flow pattern. Vega et al (2009) [8] have recently studied the fundamental importance of the convergence rate of the gas flow around the focused liquid meniscus to get the smallest possible drops. This convergence rate is mainly determined by the distance $H$ from the feeding capillary to the discharge orifice. They showed that the stability of both the tapering meniscus and the emitted jet can be critically and simultaneously met when $H \simeq D / 2$, thus 


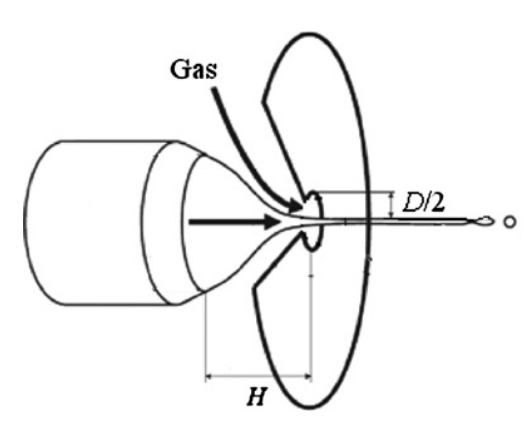

Figure 1. The steady jetting regime in the original FF configuration.

obtaining the minimum jet diameter for a given orifice. The PO geometry presents important drawbacks: (i) the sharp edges of the orifice cause strong recirculation close to the orifice surface [9], (ii) with conventional techniques (mechanical, laser, or electrochemical drilling) it is practically impossible to achieve roughnesses smaller than the boundary layer thickness $(\sim 1 \mu \mathrm{m})$ at the orifice surface and (iii) the minimum sizes of round orifices manufactured with conventional techniques are limited to tens of microns.

Nozzles are used to produce jets, drops, bubbles and other fluid shapes in very varied practical applications, such as fuel injection, ink-jet printing and sprays in industrial engineering [10, 11], or production of microcapsules [12], emulsions [13] and bubbles [14, 15] in chemical engineering and biotechnology. Micrometer nozzles have been used in microfluidics over the last few decades to obtain jets and drops by simply injecting liquids through them with the appropriate flow rate [16, 17]. The diameter of the jets and drops produced in this way is similar to that of the nozzle orifice, whose minimum value is inevitably limited to about $10 \mu \mathrm{m}$ or greater by nozzle clogging. The nearly liquid-free surface smoothness provided by flame-shaped glass nozzles reduces this risk because it makes depositions less probable. However, the problem remains at micrometer scales, the sizes of greatest interest in most modern applications.

DePonte and coworkers at Arizona State University have recently applied the FF principle [1] using a flame-polished glass micro-nozzle to produce jets of diameters much smaller than that of the nozzle orifice [18]. In this technique, a capillary of hundreds of microns in diameter is introduced into a nozzle through which a gas flows, driven by a pressure drop. Liquid at a given flow rate is injected through the capillary. If the gas pressure drop and liquid flow rate are selected appropriately, a steady conical meniscus attaches to the end of the capillary, and its tip emits a steady jet of diameter much smaller than that of the nozzle orifice. Figure 2 shows a microjet produced in our laboratory using this technique. Very recently [19], it has been shown that the tapering liquid meniscus can also be formed out of the nozzle due to the action of the co-flowing gas stream, which may entail significant technological advantages. Figure 2 also shows a SEM micrograph of a steady liquid jet obtained by this outer focusing technique. Our preliminary results indicate that this procedure requires a precisely-shaped nozzle exit.
In the present paper, we discuss the use of flame-shaped glass micro-nozzles for FF atomization [18] by showing their significant advantages over the original PO configuration. In particular, we show that focusing with nozzles is much more efficient than with their equivalent PO configurations. This is because the energy (gas) consumed by the nozzle is much smaller than that used by the corresponding PO configuration for the same gas flow pattern.

The nozzle shape plays a fundamental role in the stability of the emitted jets [18], and must satisfy some requirements to achieve outer focusing [19]. Therefore, the use of micro-nozzles in highly demanding FF applications requires measuring their three-dimensional (3D) shape precisely by non-destructive means. This fact motivated the second part of the present work, in which we propose a method to characterize the $3 \mathrm{D}$ shape of micro-nozzles formed inside millimetric and submillimetric capillaries made of transparent materials. This characterization is no trivial task, mainly due to the optical distortion caused by the capillary.

The method proposed in this paper is based on the conjunction of optical imaging and advanced image processing techniques. In optical imaging, the image of a 3D object is magnified with a microscope, and acquired and recorded by a monochrome digital camera. The silhouette effect is obtained using back-light illumination, so that the object appears in the image as an almost-black two-dimensional shape on a gray background. Advanced image processing techniques locate the contours delimiting the object with sub-pixel accuracy. The conjunction of optical imaging and advanced image processing techniques has yielded very precise results when analyzing the shape and interfacial properties of drops [20-22], bubbles [23, 24], liquid bridges [25, 26], liquid films [27, 28] and liquid lenses [29], among others. In the present work, we show that this methodology, appropriately adapted to measuring the 3D shape of glass micro-nozzles, provides precise results for nozzle sizes down to a few microns. Methods capable of measuring accurately the 3D shape of nozzles formed inside capillaries are of great interest in several fields, including biology for single cell manipulation, cell sorting, etc.

The paper is organized as follows. In section 2, we briefly describe the procedure to produce flame-shaped glass micro-nozzles for FF atomization. In section 3, we discuss the advantages of using micro-nozzles instead of the classical PO configuration. The method of nozzle geometrical characterization is described in section 4 . The characterization results are given and discussed in section 5. Some conclusions are presented in section 6 .

\section{On the fabrication of flame-shaped glass micro-nozzles}

It is well known that nozzles can be formed by applying a flame to the tip of a clean-cut millimetric glass capillary. The glass melts and begins to flow driven by the competition between surface tension and viscosity forces, both exhibiting large gradients in the heated region. This flow narrows the inner duct formed in the capillary. Before the glass inner freesurface pinches, the heat source is swiftly withdrawn, and the 


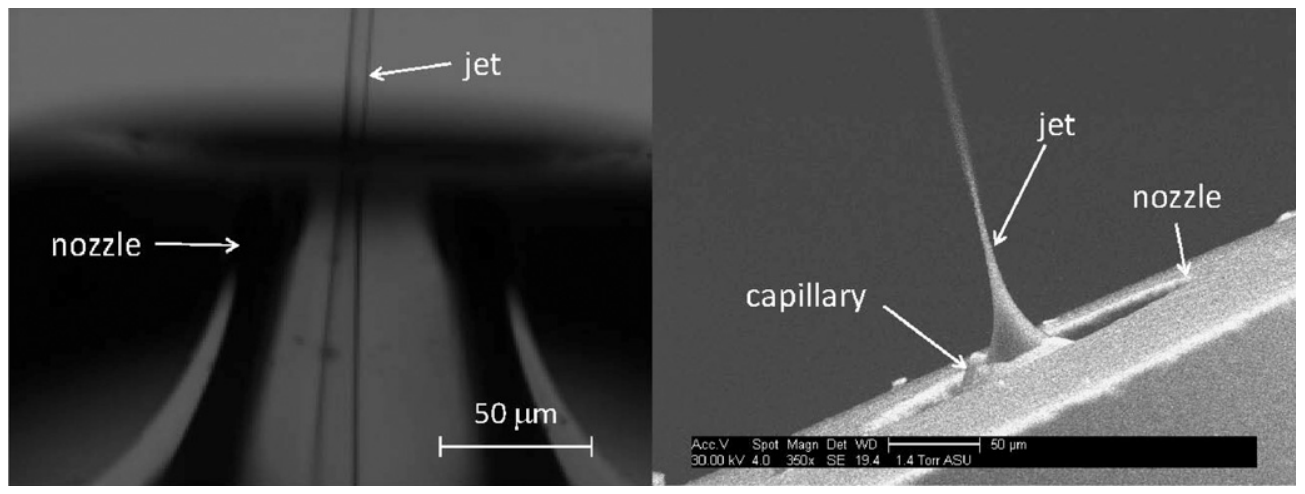

Figure 2. Left: digital image of a steady microjet emitted by a nozzle formed inside a capillary. An air stream co-flows with the liquid stretching a meniscus inside the nozzle until a microjet tapers from the menicus tip. Right: SEM micrograph recorded by DePonte and collaborators at Arizona State University of a steady tapering liquid jet [19]. The jet was emitted by a capillary introduced into a convergent nozzle. The gas stream co-flowing through the nozzle focused the liquid meniscus formed at the capillary end until a microjet was pulled out.

glass solidifies in a variety of hour-glass shapes depending on the flames intensity, focus and location. The cylindrical geometry of the primary capillary leaves the memory of the symmetry axis over the shaping process as long as the heat is applied symmetrically. Due to the large curvatures of the capillary inner free surface, the surface tension strength of glass materials (around $0.2 \mathrm{~N} \mathrm{~m}^{-1}$ ), and the smallness of the gravity effects on the melted glass, the flow keeps the nozzle symmetry down to neck sizes of a few micrometers. As a result, almost roughness-free, round orifices are obtained.

We have investigated different flame configurations and capillary-shaping procedures (with varied capillary-to-flame distances, flame application times, quenching speeds, etc.) to produce nozzle shapes with a desired convergence. Our study shows that the nozzle convergence increases as the temperature gradient in the glass increases. In order to produce nozzles with a sufficiently high degree of convergence, we applied the following procedure. A laminar propane flame was lit using a commercial gas lighter. The flame was placed so that it was just touching the capillary end. In particular, the capillary end was at the outer border of the yellow region of the flame, $4 \mathrm{~mm}$ above the lighter nozzle. The glass shaping process was observed through a microscope. This process was quenched once the desired neck diameter had been approximately reached.

Figure 3 shows two nozzles obtained with different relative positions of the capillary to the flame. When the capillary end was placed at the outer border of the flame, the temperature gradient in the glass was greater and the resulting nozzle was more convergent. This type of nozzle produces flow patterns similar to those of the optimum PO configuration, i.e. with $H \simeq D / 2$ (see the introduction). As will be shown in section 3 , these nozzles constitute a more efficient alternative to the classical PO configuration to generate the thinnest capillary jets by FF. In this way, we not only improve the original PO configuration, but also introduce an optimization procedure for the new FF embodiment conceived and developed by DePonte et al [18] at Arizona State University.

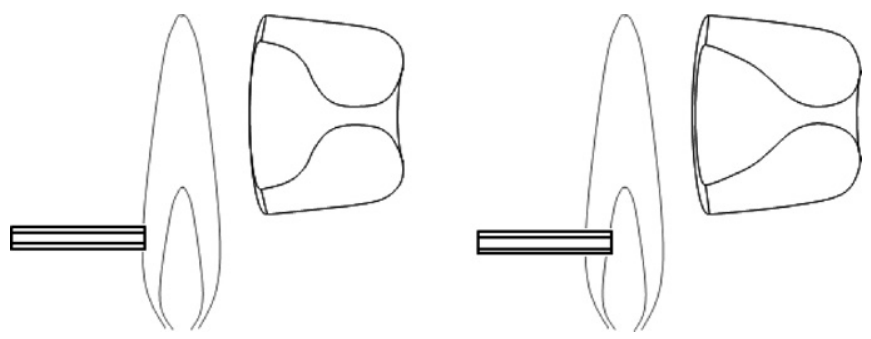

Figure 3. Nozzle shapes obtained with the indicated position of the capillary relative to the flame.

\section{Micro-nozzles versus the plate orifice configuration}

Micro-nozzles offer four major advantages over the original PO configuration: (i) they are smooth even at the micrometer scale, (ii) one can readily obtain nozzles with neck diameters in the range of tens of microns, (iii) they demand gas flow rates significantly smaller than those required by the PO configuration and (iv) they are transparent. The first three advantages are commented on in the next three subsections.

\subsection{Nozzle smoothness}

Singularities and irregularities of the solid surfaces have a negative influence on the stability of jets produced by a FF device. The sharp edges of the orifice inevitably cause strong recirculation close to the orifice surface [9], while the surface roughness may produce significant flow perturbations. These perturbations are damped by viscous forces if the surface roughness is submerged into the boundary layer which develops on the solid surface. Otherwise, the perturbations grow downstream owing to the dominance of inertia over the viscous force in the gas stream.

The gas flow induced by the pressure differences required by ultra-fine FF nebulization $[1,18]$ has characteristic speeds 
in the range from about 200 to $400 \mathrm{~m} \mathrm{~s}^{-1}$. The boundary layer developed on the nozzle surface has a characteristic thickness

$$
\delta \sim\left(\frac{\mu_{g} L}{\rho_{g} U_{g}}\right)^{1 / 2},
$$

where $\mu_{g}, \rho_{g}$ and $U_{g}$ are the characteristic gas viscosity, density and velocity at the nozzle neck, respectively, and $L$ is the characteristic nozzle length. Here, we consider the diameter at the nozzle neck as the characteristic length. If one considers the air properties and a characteristic length $L \sim 20 \mu \mathrm{m}$ (see, e.g., figures 14 and 15), then $\delta \simeq 1 \mu \mathrm{m}$. No manufacturing means other than flame polishing or isotropic chemical etching on relatively simple materials (e.g., amorphous silicon) would yield roughnesses smaller than the boundary layer thickness.

It is well known that one of the main obstacles in producing microjets and drops is the clogging of the emitting orifice. Clogging is caused by the deposition, on the orifice surface, of impurities transported by the liquid which cannot be swept away by the flow. The roughness of the solid surfaces plays a key role in the blockage of the emitting device because it makes the impurities attach to those surfaces. Therefore, the smoothness of the flame-shaped glass micro-nozzles entails an important additional advantage: it favors the removal of impurities and thus prevents the nozzle from clogging.

\subsection{Orifice and nozzle neck diameters}

FF requires a conical steady liquid meniscus to eject a microjet which ultimately breaks into droplets. The establishment of that meniscus is the result of a delicate balance between the stresses exerted on the free surface, which becomes critical close to the meniscus tip. Tangential viscous stresses $\tau_{s}$ play a fundamental role in that balance because they are essential to draw the liquid surface in the direction of the gas stream [30]. The diameter $D$ is probably the geometrical parameter that affects the balance most between the free surface stresses close to the meniscus tip. Here, $D$ is the orifice diameter in the PO configuration and the nozzle neck diameter when focusing with micro-nozzles. To analyze the effects of both the tangential viscous stresses $\tau_{s}$ and the diameter $D$ in the balance mentioned above, one can introduce the two dimensionless parameters $\tau_{s} D / \sigma$ and $D / d_{j}$, where $\sigma$ is the surface tension and $d_{j}$ is the jet diameter at the exit. A large experimental record accrued over more than a decade with the PO configuration $[30,31]$ indicates that $\tau_{s} D / \sigma \gtrsim 0.3$ and $D / d_{j} \lesssim 60$ in the FF realizations (figure 4). This last result shows that submicrometer jets and droplets can be produced with FF only if $D$ is smaller than a few tens of microns. This requirement can be readily satisfied in flame-shaped glass micro-nozzles (see e.g., figure 9), while it becomes a serious obstacle in plate orifices manufactured with conventional techniques, such as mechanical, laser and electrochemical drilling.

One of the main difficulties in using nebulizers with very small orifices is related to the starting stage. In the first instants of the injection, the liquid spreads, wets the solid surfaces and accumulates in the orifice blocking its exit. One has to remove this to properly establish a steady jetting regime. In

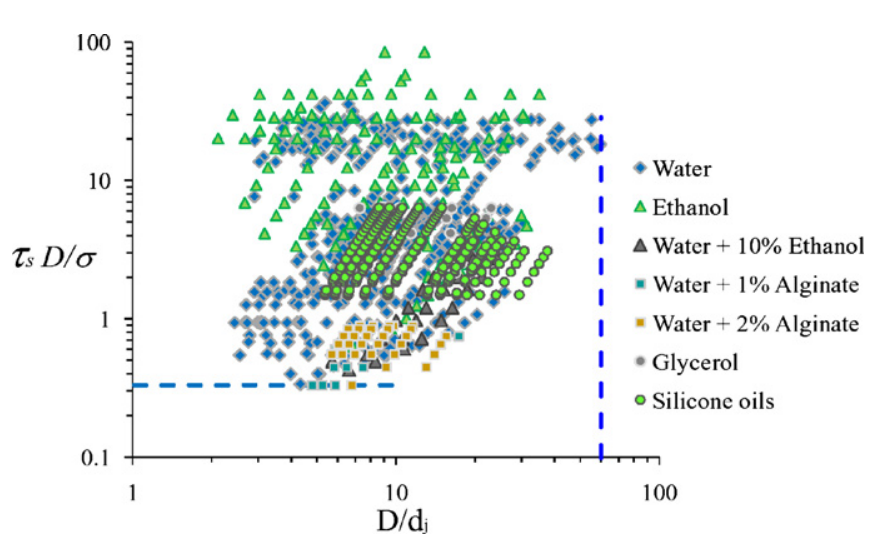

Figure 4. The 'focusing stress' parameter $\tau_{s} D / \sigma$ as a function of the ratio $D / d$.

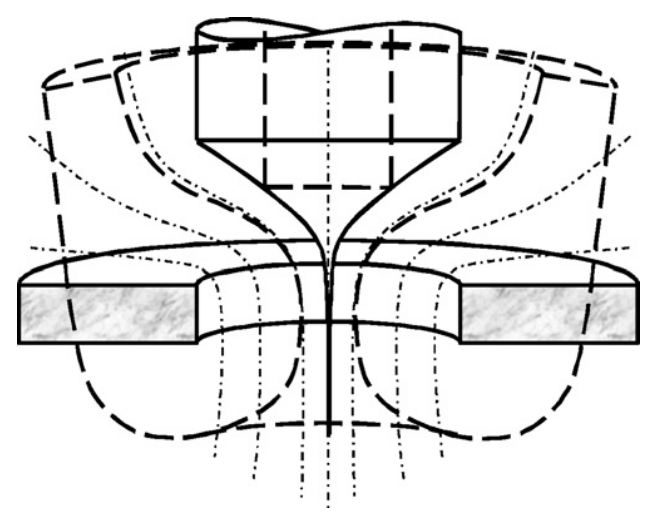

Figure 5. The optimum PO configuration and an equivalent nozzle.

FF with nozzles, this operation can be readily done by simply increasing the pressure drop applied to the gas stream in the starting stage. Due to the smoothness of the nozzle surface, the gas current easily sweeps away the deposited liquid and opens the discharge orifice. Then the pressure drop is decreased until the value corresponding to the steady regime is reached.

\subsection{Efficiency}

The gas flow in the PO configuration exhibits a streamline geometry essentially determined by the orifice diameter. This flow pattern can be generated approximately by a family of nozzle shapes with different neck diameters. Figure 5 illustrates this idea. The figure depicts the optimum $(H=$ $D / 2$ ) PO configuration [8] and an almost equivalent nozzle. The thin-dashed dotted lines are the gas streamlines while the continuous lines indicate the liquid-free surface location. The dashed lines represent the micro-nozzle which produces almost the same gas (and hence liquid) flow pattern above the neck. Note that the contour of the converging part of the nozzle practically coincides with a stream tube due to the negligible thickness (about $1 \mu \mathrm{m}$ ) of the gas boundary layers in that part of the nozzle.

The main conclusion drawn from figure 5 is that the gas (energy) consumed by the nozzle is much less than that used by the equivalent PO configuration because the nozzle neck diameter is much smaller than the orifice diameter. In other 


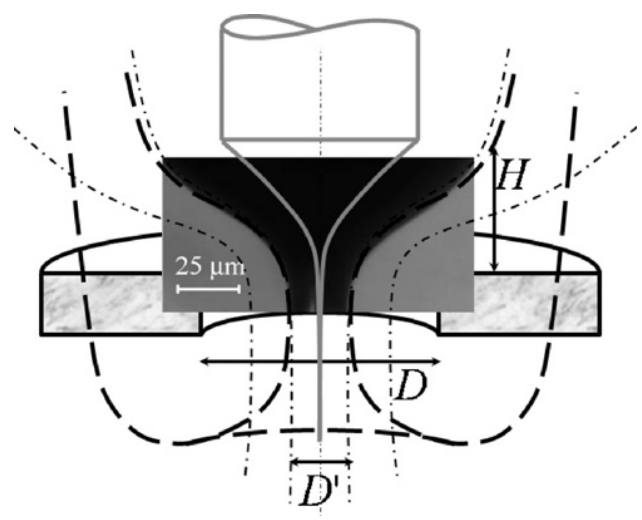

Figure 6. The nozzle analyzed in figure 14 and the corresponding PO configuration.

words, the nozzle is much more energetically efficient than the equivalent PO configuration, which may become important for massive atomization in, for instance, industrial and agricultural applications.

In principle, one could replace a given PO configuration by a nozzle nebulizer with the same focusing properties and the desired gas consumption. For this purpose, the steps to take might be the following. First, the gas stream tubes for a given PO configuration are obtained from numerical simulation [9]. Second, one calculates the diameter $D^{\prime}$ of the nozzle discharge orifice corresponding to the desired gas consumption. Finally, one fabricates a nozzle such that the contour of its converging part approximately coincides with the gas stream tube of diameter $D^{\prime}$ at a distance $H$ from the capillary. Figure 6 illustrates this procedure with one of the nozzles characterized in section 5 . The power consumed by the nozzle is $N=\Delta p\left(Q_{g}+Q_{l}\right) \simeq \Delta p Q_{g} \sim \Delta p U_{g} D^{\prime 2}$, while that consumed by the equivalent $\mathrm{PO}$ configuration is $N \sim \Delta p U_{g} D^{2}$. Here, $Q_{g}$ and $Q_{l}\left(Q_{l} \ll Q_{g}\right)$ are the gas and liquid flow rates, respectively, $\Delta p$ is the pressure drop applied to the gas stream and energy dissipation in the gas and liquid injection systems has been neglected.

In contrast to what happens with the classical PO configuration, the production of appropriate micro-nozzles for FF atomization requires a precise method of characterizing their internal shape. In the next section, we propose a nondestructive technique to obtain the 3D shape of micro-nozzles formed inside capillaries made of transparent materials.

\section{Method of geometrical characterization}

\subsection{Apparatus}

Figure 7 shows the main components of the apparatus used to characterize the nozzles. The nozzle formed inside a capillary (A) made of borosilicate (haematocrit glass capillary with $\mathrm{OD}=1.55 \mathrm{~mm}$ and $\mathrm{ID}=1.15 \mathrm{~mm}$ ) was put in a cubic glycerin (with refractive index $n=1.474$ ) bath (B), which had almost the same refractive index as that of borosilicate ( $n$ from 1.47 to 1.5). The capillary was held onto a precision rotation platform (C) to rotate the capillary around a vertical axis. Digital images of the capillary consisting of $1292 \times 964$ pixels were acquired

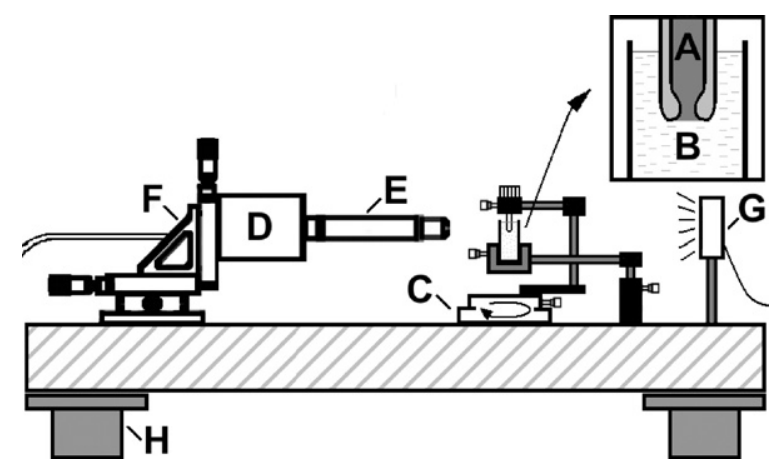

Figure 7. Experimental apparatus: nozzle (A), glycerin bath (B), precision rotation platform $(C)$, camera $(D)$, optical lenses $(E)$, triaxial translation stage $(\mathrm{F})$, back-lighting $(\mathrm{G})$ and optical table $(\mathrm{H})$.

with an AVT STINGRAY F-125B CCD camera (D) equipped with optical lenses (a MITUTOYo 10× magnification zoom-objective and an OPTEM 70XL set of lenses with variable magnification from $0.75 \times$ to $5.25 \times)(\mathrm{E})$ providing a variable frame covering an area from $672 \times 502 \mu \mathrm{m}$ down to $97.7 \times 72.9 \mu \mathrm{m}$. The resulting magnification could be selected within an interval of approximately between 520 and $75.6 \mathrm{~nm} /$ pixel. The camera could be displaced by means of a high-precision triaxial translation stage (F) to focus the capillary. The capillary was illuminated using back-light illumination $(\mathrm{G})$ to get a silhouette effect, so that the inner contour of the capillary (the nozzle) appeared in the image as a black two-dimensional object on a gray background. All the components of the apparatus were mounted on an optical table with a pneumatic anti-vibration isolation system $(\mathrm{H})$ to damp the vibrations coming from the building.

\subsection{Procedure}

Before characterizing the nozzles, the image acquisition system was calibrated. For this purpose, digital images of calibration rods with different diameters were acquired and analyzed to calculate the magnifications used subsequently. We also acquired and processed images of a calibration grid using lenses with less magnification to verify that the pixel aspect ratio was practically unity. The optical distortion associated with the optical lenses was assumed to be negligible as compared to that caused by the borosilicate capillary/glycerin bath set.

The procedure to characterize a nozzle consisted of the following steps.

(i) The inner wall of the capillary (the nozzle) was colored by injecting ink and dried with an infrared lamp.

(ii) The capillary was put in the glycerin bath to partially remove the optical distortion caused by the capillary. Figure 8 shows an image of the nozzle before (a) and after (b) coloring and putting it in the glycerin. The two images were acquired with the same optical system, and thus they correspond to almost the same field of view. The capillary distorted the apparent shape of the nozzle in such a way that the nozzle looked a converging duct while actually it had a diverging part. 

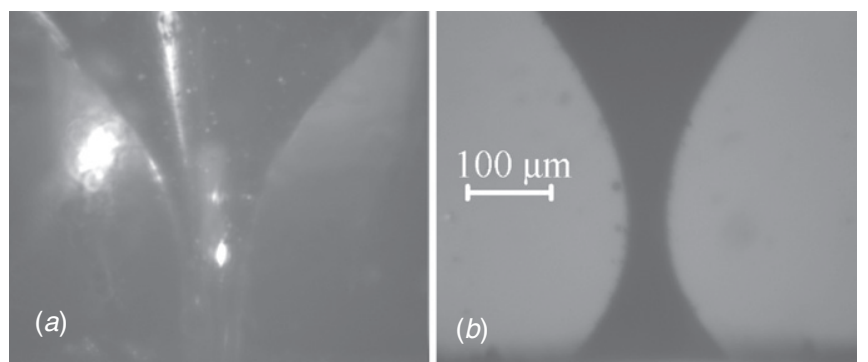

Figure 8. Digital image of the nozzle before $(a)$ and after $(b)$ coloring and dipping in glycerin. The two images were acquired with the same optical system, and thus they correspond to the same field of view.

(iii) For a given position of the rotation platform, a digital image of the capillary in the glycerin bath was acquired and recorded.

(iv) The capillary was rotated $9^{\circ}$ around a vertical axis using the precision rotation platform.

Steps (iii) and (iv) were repeated 180/9 $=20$ times to get images of the 20 meridional planes used to compose the $3 \mathrm{D}$ shape of the nozzle.

It must be noted that the capillaries could be easily cleaned to re-use them after characterizing the shape of the nozzles. When the size of the nozzle was sufficiently small (say less than $100 \mu \mathrm{m}$ ), it was not necessary to color the inner wall of the capillary to get the silhouette effect (see figures 14 and 15), which simplified the characterization process.

\subsection{Image processing technique}

Following the procedure described in section 4.2, 20 images of the nozzle to be characterized are acquired and recorded. Each of those images shows the silhouette of the nozzle meridional plane brought face to face with the camera. The meridional plane appears in the image as an almost black object on a gray background. Advanced image processing techniques have been designed to locate precisely the contours delimiting the objects in this type of images. They provide results with sub-pixel accuracy when processing images of drops [20-22], bubbles [23, 24], liquid bridges [25, 26], liquid films [27, 28] and liquid lenses [29], among others. In the present work, we used an image processing technique recently proposed to locate the free surface enclosing liquid shapes of tens of micrometers in size [28, 32]. This technique is capable of 'restoring' blurred edges to partially overcome limitations due, for instance, to diffraction or lack of focus. For the sake of completeness, we describe in this section the main aspects of that technique.

4.3.1. Detection of the contours. In our experiments, a digital image is defined mathematically by the gray intensity matrix $I(i, j)$, which takes an integer value between 0 (black) and 255 (white) for each pixel $(i, j)$, where $i$ and $j$ correspond to the horizontal and vertical directions, respectively. The nozzle contours must correspond to a step in the gray intensity in the direction perpendicular to those contours. However,

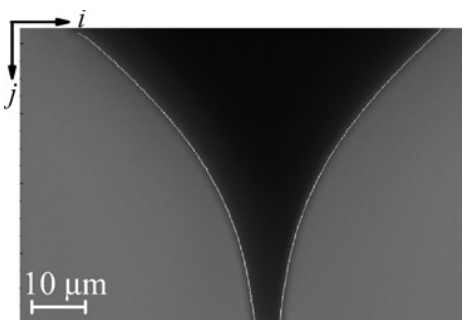

Figure 9. Digital image of the nozzle and the contours (white lines) detected at the pixel level with the Otsu method.

the intensity change is smeared out over several pixels, and determining the position of the contours is not obvious.

In the present work, the nozzle contours were detected using a two-stage procedure. In the first stage, a set of pixels $\left\{\left(i_{c}, j_{c}\right)\right\}$ probably corresponding to the contours being sought was extracted using Otsu's method [33]. In this method, a threshold value of the gray intensity is calculated by assuming that the histogram of $I(i, j)$ is a combination of two distributions corresponding to the object and the background. The threshold value separates those two distributions in the histogram and is calculated by minimizing the weighted sum of their variances. More details of the Otsu method can be found in the original work [33]. Figure 9 shows a digital image of the nozzle and the contours (white lines) detected at the pixel level with the Otsu method.

The accuracy of Otsu's method is limited to the pixel size. In the second stage, the local intensity threshold criterion is used at the sub-pixel level to improve the precision of the result. For every pixel $\left(i_{c}, j_{c}\right)$, the direction $\xi$ normal to the contour is determined as that of the intensity gradient. To calculate the intensity gradient, an operator such as the $3 \times 3$ Sobel convolution kernel can be applied to the gray intensity matrix $I(i, j)$ previously smoothed with a filter, such as the Gaussian filter. It must be noted that the use of the horizontal axis $i$ instead of the normal direction $\xi$ in the subsequent calculations yields very similar results and reduces the computation time significantly.

Once the $\xi$ direction has been determined for every contour pixel $\left(i_{c}, j_{c}\right)$, the sigmoid (Boltzmann) function [20]

$$
g(\xi)=\frac{g_{1}-g_{2}}{1+\exp \left[\left(\xi-\xi_{0}\right) / W\right]}+g_{2}
$$

is fitted to the gray intensity profile along the $\xi$ axis. Here, $g_{1}$ and $g_{2}$ correspond to the plateau gray values at the two sides of the edge, $W$ is a measure of the edge width, and $\xi_{0}$ is the midpoint of the profile. The contour point is given by $\xi_{0}$, for which (2) takes the value $\left(g_{1}+g_{2}\right) / 2$, and thus the method can be seen as a local thresholding technique. The right-hand graph in figure 10 shows the gray intensity profile (symbols) along the dashed line indicated in the image and the corresponding sigmoid function fits (solid lines). The vertical dashed lines indicate the positions of the left and right contours, where (2) takes the corresponding local threshold values $\left(g_{1}+g_{2}\right) / 2$. When this procedure is applied to each pixel $\left(i_{c}, j_{c}\right)$, the result is two sets of points $\left\{\left(x_{l}, z\right)\right\}$ and $\left\{\left(x_{r}, z\right)\right\}$ representing the left and right nozzle contours, respectively. In our calculations, if the distance between the pixel $\left(i_{c}, j_{c}\right)$ and the corresponding 

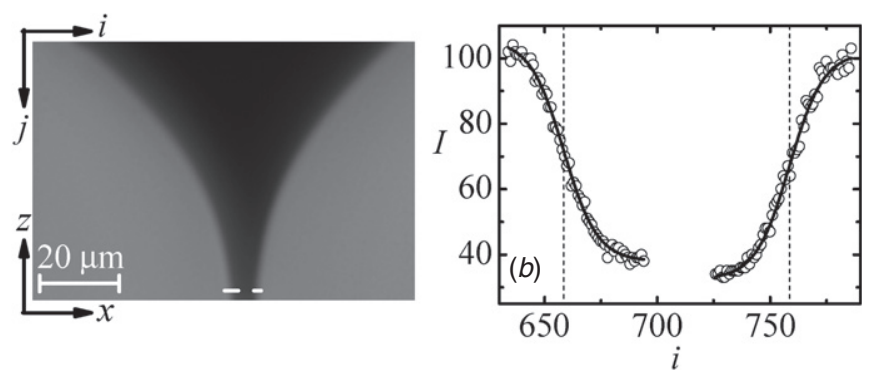

Figure 10. Left: digital image of the nozzle. Right: the gray intensity profile (symbols) along the dashed line indicated in the image, and the corresponding sigmoid function fits (solid lines). The vertical dashed lines indicate the positions of the left and right contours.

point calculated at the sub-pixel level is greater than twice the pixel size, then that point is rejected. The sub-pixel approach significantly improves the results obtained at the pixel level for most images. In particular, it improves the precision of the contours detected in sharp images [20-22, 25-27, 29, 34], and allows one to filter anomalous points in low-quality images $[23,24]$.

It must be noted that the present technique constitutes a useful alternative to more standard sub-pixel resolution methods (see e.g. [22, 25-27]) under several circumstances. Those methods generally calculate the local threshold of the gray intensity profile as the average of the two plateaus existing at each side of the profile, and then determine the contour point by interpolating the gray profile in the transient region. The present technique has two main advantages with respect to that procedure: (i) while that procedure only filters the noise on the two sides of the edge to calculate the gray intensity threshold, fitting (2) to the entire gray intensity profile filters the noise in the transient region too; and (ii) fitting (2) to the gray intensity profile provides accurate results even when the distance between the left and right contours is so short that the gray intensity profiles overlap, making it difficult to identify the plateaus in the interference region. Indeed, this is the case shown in figure 10, for which standard techniques do not provide satisfactory results.

\subsubsection{Calculation of the axisymmetric contour. As} mentioned in section 2, the necks of the nozzles formed inside glass capillaries generally have large curvatures, and thus their shapes are nearly axisymmetric. In the present work, the symmetry axis $x_{s}(z)$ of the nozzle neck was found by an elemental procedure, and the left $x_{l}(z)$ and right $x_{r}(z)$ contours were rotated until $x_{s}(z)$ was almost vertical. Then, the lack of symmetry of the entire nozzle was evaluated by calculating the eccentricity $E(z) \equiv\left|x_{l}^{*}(z)-x_{s}\right|-\left|x_{r}^{*}(z)-x_{s}\right|$, where $x_{l}^{*}(z)$ and $x_{r}^{*}(z)$ are the left- and right-rotated contours, respectively. The eccentricity $E(z)$ can be seen as a quality parameter which indicates the degree of uniformity of the heating used to form the nozzle. In addition, an 'average' axisymmetric contour was obtained from the left- and rightrotated contours as $F^{*}=\left(x_{l}^{*}-x_{r}^{*}\right) / 2$.

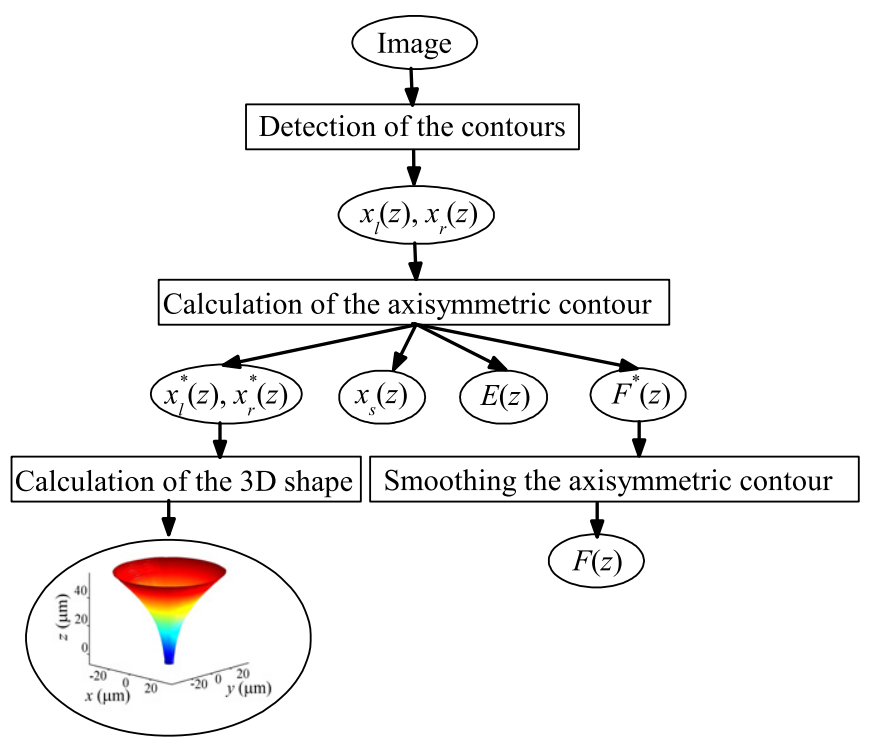

Figure 11. Flow chart showing the stages of the image processing technique and the quantities obtained.

4.3.3. Smoothing the axisymmetric contour. The method described in sections 4.3.1 and 4.3.2, as applied to our images, provides a set of points $\left\{\left(F^{*}, z\right)\right\}$ representing the nozzle contour. Because this set must belong to a smooth function, smoothing techniques can be used to improve the result. An elemental smoothing algorithm was used to reduce the fluctuations of the contour position. It is similar to that used in $[23,35]$ to smooth the free surface position of pendant drops to measure the surface tension. It proceeds as follows. Consider a point $\left(F^{*}, z\right)$ belonging to the nozzle contour. $2 M+1$ contiguous points of the contour are taken around $\left(F^{*}, z\right)$. An $O$ th-order polynomial fit to that set of points is calculated. The original central point $\left(F^{*}, z\right)$ is replaced with that obtained from the fit $(F, z)$. The smoothed contours $\{(F, z)\}$ to be presented in section 5.1 were obtained by applying this procedure with $M=40$ and $O=3$ to the original contour points $\left\{\left(F^{*}, z\right)\right\}$.

\subsection{Calculation of the three-dimensional shape}

As explained in section 4.2, 20 images corresponding to an equal number of nozzle meridional planes were acquired and recorded in the measuring process. Following the procedure described in sections 4.3.1 and 4.3.2, the left and right contours delimiting those meridional planes were determined and rotated to the vertical position.

In order to obtain the 3D shape of the nozzle, the rotated contours $x_{l}^{*}(z)$ and $x_{r}^{*}(z)$ must be referred to a common system of coordinates. As mentioned in the previous section, the nozzles analyzed were almost axisymmetric in the region close to their necks. Therefore, the symmetry axes calculated from that region of the meridional planes must coincide. In addition, the height $z$ corresponding to the minimum width of the nozzle must also be the same for all the images of the nozzle. We used these two conditions to refer the contours $x_{r}^{*}(z)$ and $x_{l}^{*}(z)$ of the 20 images to a common coordinate system. In this way, the set of points in the $3 \mathrm{D}$ space belonging to the nozzle contour 

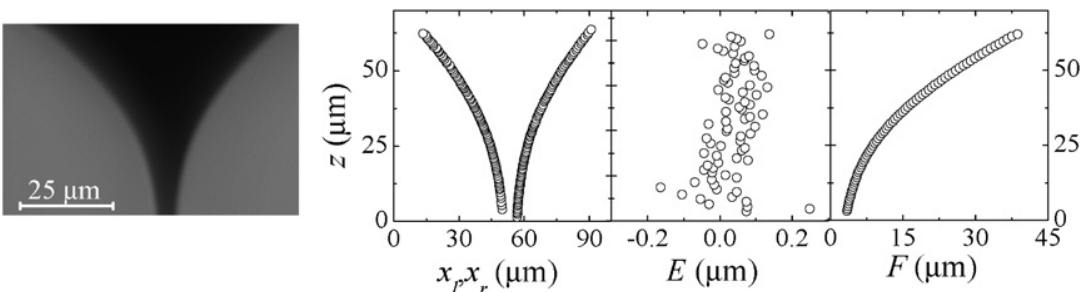

Figure 12. Left $x_{l}(z)$ and right $x_{r}(z)$ nozzle contours, eccentricity $E(z)$, and axisymmetric contour $F(z)$ corresponding to the digital image shown in the figure.
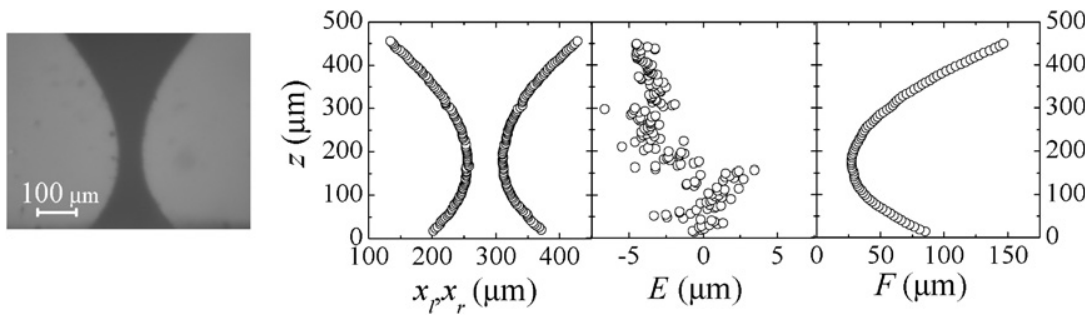

Figure 13. Left $x_{l}(z)$ and right $x_{r}(z)$ nozzle contours, eccentricity $E(z)$, and axisymmetric contour $F(z)$ corresponding to the digital image shown in the figure.
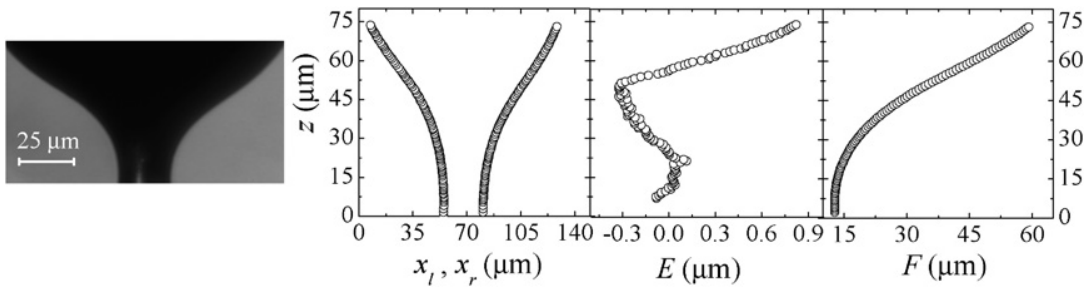

Figure 14. Left $x_{l}(z)$ and right $x_{r}(z)$ nozzle contours, eccentricity $E(z)$, and axisymmetric contour $F(z)$ corresponding to the digital image shown in the figure.
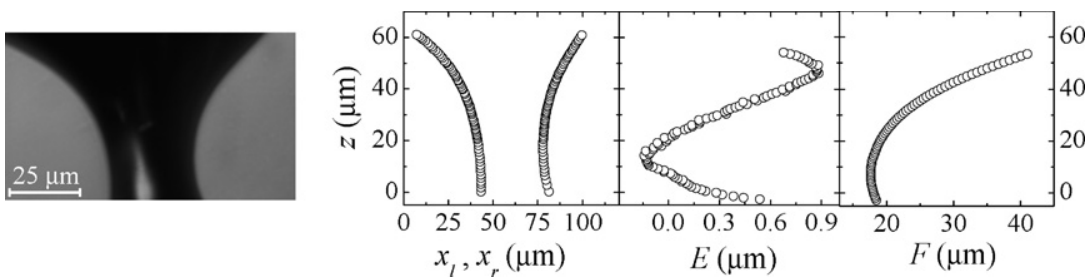

Figure 15. Left $x_{l}(z)$ and right $x_{r}(z)$ nozzle contours, eccentricity $E(z)$, and axisymmetric contour $F(z)$ corresponding to the digital image shown in the figure.

could be obtained easily. The final result was a continuous surface calculated by interpolating that set of points.

Figure 11 is a flow chart showing the stages of the image processing technique and the quantities obtained in the analysis.

\section{Characterization results}

In order to illustrate the capabilities of the method proposed in this paper, we show in this section the results obtained for several nozzles. Figures 12-15 show the left $x_{l}(z)$ and right $x_{r}(z)$ nozzle contours, the nozzle eccentricity $E(z)$ and the smoothed axisymmetric shape $F(z)$ calculated for a meridional plane of four nozzles, while the 3D shape of two nozzles is plotted in figure 16. The nozzles analyzed in figures 12, 13 and 16 were manufactured in our laboratory, while figures 14 and 15 show the results for two custom tip type II nozzles manufactured by Eppendorf AG.

\subsection{Two-dimensional (axisymmetric) results}

Figure 12 shows the shape of a highly converging nozzle with a neck radius smaller than $5 \mu \mathrm{m}$. The eccentricity had no spatial structure and was less than $200 \mathrm{~nm}$ over the entire section analyzed, which reflects the high degree of symmetry achieved in the manufacturing process. A very smooth contour $F(z)$ was finally obtained after processing the image.

Figure 13 shows the shape of a converging-diverging nozzle with a neck radius of about $30 \mu \mathrm{m}$. The eccentricity had hardly any spatial structure and was smaller than $5 \mu \mathrm{m}$, 

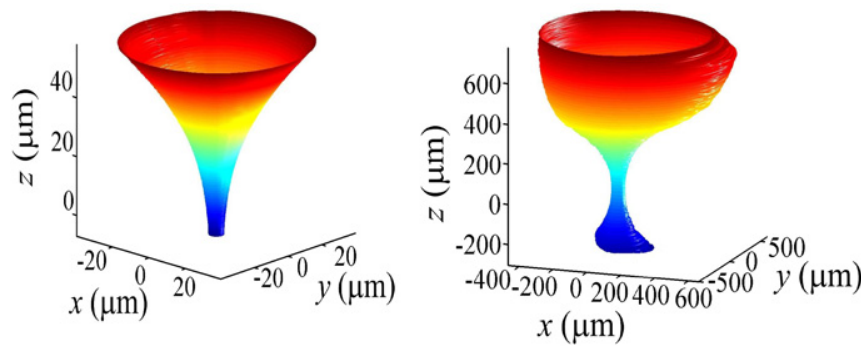

Figure 16. 3D results obtained for an axisymmetric (left) and defective (right) nozzle.

less than $2 \%$ of the maximum diameter. In the image, one can appreciate the existence of small blotches close to the nozzle contour. They probably correspond to bubbles formed in the melting process and trapped when the glass solidified. These stains constituted an obstacle for accurate detection of the nozzle contours. Nevertheless, the image processing technique overcame this difficulty and still provided a smooth axisymmetric contour $F(z)$.

In figures 14 and 15, we plot the results obtained for two nozzles without coloring them. As mentioned in section 4.2, back-light illumination produced the silhouette effect for very small nozzles by simply putting them in the glycerin bath. The resulting images were sharp and could be acquired and accurately processed in a few minutes. The spatial structure of the eccentricity revealed a certain lack of symmetry of the nozzles.

\subsection{Three-dimensional results}

As explained in section 4.4 , the 3D shape of the nozzle can be visualized by combining different meridional planes. By way of illustration, figure 16 shows the results obtained for an axisymmetric (left) and defective (right) nozzle. In the latter case, non-uniform heating caused noticeable asymmetries far from the neck, where the effect of surface tension was less important.

Establishing the accuracy of the results presented in this section is a very hard task even when resorting to a destructive technique. In this case, one would firstly cut away the outer wall of the glass capillary until making the nozzle meridional cross-section visible. It can be easily verified that the distance $\Delta$ between the nozzle meridional plane and the front plane obtained after polishing the capillary cut would be $\Delta \simeq\left(2 F\left|F-F^{*}\right|\right)^{1 / 2}$, where $F^{*}$ would be the apparent nozzle contour exhibited (figure 17). We will assume that the error assigned to our characterization method is of the order of 1 pixel size, i.e. $0.1 \mu \mathrm{m}$ in most of the images (see section 4.1). If $F \sim 10 \mu \mathrm{m}$ (see figures 12,14 , and 15), then $\Delta$ must be smaller than $(2 \times 10 \times 0.1)^{1 / 2}=1.41 \mu \mathrm{m}$. Otherwise, the validation method would lead to errors larger than those of our method. Unfortunately, we do not have access to such a glass polishing technique to achieve the required precision.

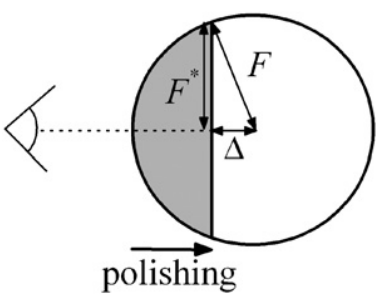

Figure 17. The distance $\Delta$ between the nozzle meridional plane and the front plane obtained after polishing the capillary.

\section{Conclusions}

In this paper, we have discussed in detail a new configuration for capillary FF using flame-shaped glass micro-nozzles [18]. The main advantages offered by these nozzles over the classical PO configuration [1] are: (i) they are extraordinarily smooth even at the micrometer scale, (ii) one can readily obtain nozzles with neck diameters in the range of a few tens of microns, (iii) they demand gas flow rates (energy) significantly smaller than those required by the PO configuration and (iv) they are transparent. However, highly demanding applications require a precise characterization of their 3D shape by means of nondestructive techniques. In contrast to the case with the classical PO configuration, this characterization is no trivial task, mainly due to the optical distortion caused by the capillary. For the purposes of this quantitative characterization, we have proposed a non-destructive method to measure the 3D shape of micro-nozzles formed inside capillaries made of transparent materials. The method is based on the conjunction of optical imaging and advanced image processing techniques. Section 5 shows the results obtained for several nozzles. The contours delimiting the meridional planes of the nozzles analyzed were precisely determined. Submicrometer fluctuations were obtained in those contours for all the cases considered.

Also, the microscopic characterization method described in this paper can be used in very varied applications. Specifically, it can be used to determine the shape of very small channels used in microfluidics, in which many of the technologies used to produce microjets, drops, capsules and bubbles require nozzles from tens to hundreds of microns in size, and the role played by the nozzle shape may become critical to attain satisfactory results.

\section{Acknowledgments}

This research was supported by the Ministerio de Educación y Ciencia (Spain) through grant no DPI2007-63559. Partial support from the Junta de Extremadura through grant no GRU07003 is also acknowledged. We are very grateful to Dr Conrado Ferrera for discussions and experimental assistance.

\section{References}

[1] Gañán-Calvo A M 1998 Generation of steady liquid microthreads and micron-sized monodisperse sprays in gas streams Phys. Rev. Lett. $80285-8$ 
[2] Basaran O A 2002 Small-scale free surface flows with breakup: drop formation and emerging applications $A l C h E$ J. 48 1842-8

[3] Eggers J and Villermaux E 2008 Physics of liquid jets Rep. Prog. Phys. 71036601

[4] Plateau J 1849 Sur les figures d'equilibre d'une masse liquide sans pesanteur Mem. Acad. R. Belg. Nouv. Ser. 23

[5] Rayleigh L 1879 On the instability of jets Proc. Lond. Math. Soc. $104-13$

[6] Anna S L, Bontoux N and Stone H A 2003 Formation of dispersions using flow focusing in microchannels Appl. Phys. Lett. 82 364-6

[7] Anna S L and Mayer H C 2006 Microscale tipstreaming in a microfluidic flow focusing device Phys. Fluids 18121512

[8] Vega E J, Montanero J M, Herrada M A and Gañán-Calvo A M 2010 Global and local instability of flow focusing: the influence of the geometry Phys. Fluids at press

[9] Herrada M A, Gañán-Calvo A M, Ojeda Monge A, Bluth B and Riesco-Chueca P 2008 Liquid flow focused by a gas: jetting, dripping, and recirculation Phys. Rev. E 78036323

[10] Okeefe J D, Wrinkle W W and Scully C N 1967 Supersonic liquid jets Nature 7 23-5

[11] Yarin A L 2006 Drop impact dynamics: splashing, spreading, receding, bouncing. Annu. Rev. Fluid Mech. 38 159-92

[12] Gañán-Calvo A M, González-Prieto R, Riesco-Chueca P, Herrada M A and Flores-Mosquera M 2007 Focusing capillary jets close to the continuum limit Nat. Phys. $3737-42$

[13] Utada A S, Lorenceau E, Link D R, Kaplan P D, Stone H A and Weitz D A 2005 Monodisperse double emulsions generated from a microcapillary device Science 308 537-41

[14] Gañán Calvo A M and Gordillo J M 2001 Perfectly monodisperse microbubbling by capillary flow focusing Phys. Rev. Lett. 87274501

[15] Stride E and Edirisinghe M 2008 Novel microbubble preparation technologies Soft Matter 4 2350-9

[16] Switzer G L 1991 A versatile system for stable generation of uniform droplets Rev. Sci. Instrum. 62 2765-71

[17] Weierstall U, Doak R B, Spence J C H, Starodub D, Shapiro D, Kennedy P, Warner J, Hembree G G, Fromme P and Chapman H N 2008 Droplet streams for serial crystallography of proteins Exp. Fluids 44 675-89

[18] DePonte D P, Weierstall U, Schmidt K, Warner J, Starodub D, Spence J C H and Doak R B 2008 Gas dynamic virtual nozzle for generation of microscopic droplet streams J. Phys. D: Appl. Phys. 41195505

[19] Gañán-Calvo A M, DePonte D P, Herrada M A, Spence J C H, Weierstall U and Doak R B 2010 Liquid capillary micro/nanojets in free-jet expansion Small 6 822-4
[20] Song B and Springer J 1996 Determination of interfacial tension from the profile of a pendant drop using computer-aided image processing: 2. Experimental J. Colloid Interface Sci. 184 77-91

[21] Cabezas M G, Bateni A, Montanero J M and Neumann A W 2005 A new method of image processing in the analysis of axisymmetric drop shapes Colloids Surf. A 255 193-200

[22] Hoorfar M and Neumann A W 2006 Recent progress in axisymmetric drop shape analysis (ADSA) Adv. Colloid Interface Sci. 121 25-49

[23] Zuo Y Y, Ding M, Bateni A, Hoorfar H and Neumann A W 2004 Improvement of interfacial tension measurement using a captive bubble in conjunction with axisymmetric drop shape analysis (ADSA) Colloids Surf. 62 233-46

[24] Zuo Y Y, Chau D and Neumann A W 2007 Automatic measurement of surface tension from noisy images using a component labeling method Colloids Surf. A 299 109-16

[25] Lowry B J 1996 Pressure and stress measurement via image analysis (P-SIA) of axisymmetric drops and liquid bridges J. Colloid Interface Sci. 176 284-97

[26] Ferrera C, Cabezas M G and Montanero J M 2006 An experimental analysis of the linear vibration of axisymmetric liquid bridges Phys. Fluids 18082105

[27] Tavana H and Neumann A W 2007 Recent progress in the determination of solid surface tensions from contact angles Adv. Colloid Interface Sci. 132 1-32

[28] Montanero J M, Vega E J and Ferrera C 2009 Sub-micrometer precision of optical imaging to locate the free surface of a micrometer fluid shape J. Colloid Interface Sci. 339 271-4

[29] Cabezas M G, Bateni A, Montanero J M and Neumann A W 2006 Determination of surface tension and contact angle from the shapes of axisymmetric fluid interfaces without use of apex coordinates Langmuir 22 10053-60

[30] Gañán-Calvo A M and Montanero J M 2009 Revision of capillary cone-jet physics: electrospray and flow focusing Phys. Rev. E 79066305

[31] Rosell-Llompart J and Gañán-Calvo A M 2008 Turbulence in pneumatic flow focusing and flow blurring regimes Phys. Rev. E 77036321

[32] Montanero J M, Vega E J and Ferrera C 2009 Sub-micrometer precision of optical imaging to locate the free surface of a micrometer fluid shape J. Colloid Interface Sci. 339 271-4

[33] Otsu N 1979 A threshold selection method from gray-level histograms IEEE Transactions on Systems, Man and Cybernetics

[34] Vega E J, Montanero J M and Fernández J 2009 On the precision of optical imaging to study free surface dynamics at high frame rates Exp. Fluids 47 251-61

[35] Anastasiadis S H, Chen J K, Koberstein J T, Siegel A F, Sohn J E and Emerson J A 1987 The determination of interfacial tension by video image processing of pendant fluid drops J. Colloid Interface Sci. 119 55-66 